\title{
Continuity of Gevrey-Hörmander pseudo-differential operators on modulation spaces
}

\section{Joachim Toft ${ }^{1}$}

Received: 1 October 2018 / Accepted: 17 December 2018 / Published online: 17 January 2019

(c) The Author(s) 2019

\section{Abstract}

Let $s \geq 1, \omega, \omega_{0} \in \mathscr{P}_{E, s}^{0}, a \in \Gamma_{s}^{\left(\omega_{0}\right)}$, and let $\mathscr{B}$ be a suitable invariant quasiBanach function space. Then we prove that the pseudo-differential operator $\operatorname{Op}(a)$ is continuous from $M\left(\omega_{0} \omega, \mathscr{B}\right)$ to $M(\omega, \mathscr{B})$.

Keywords Pseudo-differential operators · Modulation spaces · BF-spaces · Gelfand-Shilov spaces

Mathematics Subject Classification 35S05 - 47B37 · 47G30 • 42B35

\section{Introduction}

Several problems in physics, engineering, partial differential equations, timefrequency analysis and signal processing are ill-posed in the framework of the classical function and distribution spaces

$$
C_{0}^{\infty}, \quad \mathscr{S}, \quad C^{\infty}, \quad \mathscr{D}^{\prime}, \quad \mathscr{S}^{\prime}, \quad \text { and } \mathscr{E}^{\prime}
$$

(see [21] or Sect. 2 for notations). For example, the Euler-Tricomi equation $D_{t}^{2} f+$ $t D_{x}^{2} f=0$, useful in the study of transonic flow, is ill-posed in such classical setting. On the other hand, the Euler-Tricomi equation is well-posed in the framework of suitable Gelfand-Shilov or Gevrey spaces and their spaces of ultra-distributions in place of (1.1). (See [4,26].)

An other classical example concerns the heat problem

$$
\partial_{t} f=\Delta_{x} f, \quad f(0, x)=f_{0}(x), \quad t \in \mathbf{R}, \quad x \in \Omega,
$$

$凶$ Joachim Toft

joachim.toft@lnu.se

1 Department of Mathematics, Linnæus University, Växjö, Sweden

Dirkhäuser 
where $\Omega$ is a cuboid. It is well-posed when moving forward in time ( $t>0$ ), but ill-posed when moving backwards in time $(t<0)$ within the framework of classical function and distribution spaces. On the other hand, by [37, Example 2.16] it follows that the heat problem is well-posed for suitable Gelfand-Shilov distribution spaces and Gevrey classes when $t<0$. Furthermore, if $t>0$, then more precise continuity descriptions is deduced in the framework of such spaces compared to classical function and distribution spaces.

Pseudo-differential operators appear in natural and several ways when dealing with problems in partial differential equations, e.g. at above. As long as the analyses for such equations stays within the usual functions and distribution spaces, the symbols to related pseudo-differential operators often belong to the classical symbol classes, given in [21]. On the other hand, when discussing problems within Gelfand-Shilov spaces of functions and distributions, the conditions on the symbols need to be modified, to meet the stronger regularity in corresponding test function spaces.

In the paper we consider continuity properties for a class of pseudo-differential operators introduced in [5] when acting on a broad class of modulation spaces, given in $[11,12,15]$. The symbols of the pseudo-differential operators are smooth, should obey strong ultra-regularity of Gevrey or Gelfand-Shilov types, and are allowed to grow exponentially or subexponentially.

More precisely, we consider pseudo-differential operators with symbols in the spaces $\Gamma_{s}^{\left(\omega_{0}\right)}$ or in $\Gamma_{0, s}^{\left(\omega_{0}\right)}$ of Gevrey types which consist of all smooth $a$ defined on the phase space such that

$$
\left|\partial^{\alpha} a\right| \leq C h^{|\alpha|} \alpha !^{s} \omega_{0}
$$

holds for some constant $h>0$ or every constant $h>0$, respectively. Here $\omega_{0}$ is a suitable weight on the phase space and the constant $C>0$ is only depending on $h$. In [5] it is proved that if $\omega_{0} \lesssim e^{r|\cdot|^{\frac{1}{s}}}$ for some $r>0$ and $a \in \Gamma_{0, s}^{\left(\omega_{0}\right)}$, then corresponding pseudo-differential operators $\operatorname{Op}(a)$ is continuous on the Gelfand-Shilov space $\Sigma_{S}$ of Beurling type, and its distribution space $\Sigma_{s}^{\prime}$. If instead $\omega_{0} \lesssim e^{r|\cdot|^{\frac{1}{s}}}$ holds for every $r>0$ and $a \in \Gamma_{s}^{\left(\omega_{0}\right)}$, then $\operatorname{Op}(a)$ is continuous on the Gelfand-Shilov space $\mathcal{S}_{S}$ of Roumieu type, and its distribution space $\mathcal{S}_{S}^{\prime}$ (Cf. Theorems 4.10 and 4.11 in [5]).

In Sect. 3 we enlarge this family of continuity results by deducing continuity properties for such pseudo-differential operators when acting on a broad family of modulation spaces. More precisely, let $\omega_{0}$ be as above, and suppose that the weight $\omega$ satisfies

$$
0<\omega(X+Y) \lesssim \omega(X) e^{r|Y|^{\frac{1}{s}}}, \quad X, Y \in \mathbf{R}^{2 d}
$$

for every $r>0$ when $a \in \Gamma_{s}^{(\omega)}$ and satisfies (1.3) for some $r>0$ when $a \in \Gamma_{0, s}^{(\omega)}$. Also let $\mathscr{B}$ be a suitable invariant quasi-Banach-function space (QBF-space) and let $M(\omega, \mathscr{B})$ be the modulation space with respect to $\omega$ and $\mathscr{B}$. Then we prove that $\mathrm{Op}(a)$ is continuous from $M\left(\omega_{0} \omega, \mathscr{B}\right)$ to $M(\omega, \mathscr{B})$ (cf. Theorems $3.5,3.8,3.10$ and Corollary 3.11$)$. In the case when $\mathscr{B}$ is a Banach space, then the restrictions on $\mathscr{B}$ 
are given in Definition 2.2, while if $\mathscr{B}$ fails to be a Banach space, then suitable mixed Lebesgue quasi-norm estimates are imposed on the elements in $\mathscr{B}$.

Certain Gelfand-Shilov spaces and their distribution spaces are equal to suitable intersections and unions of modulation spaces (see e.g. [33,35]). This implies that the continuity properties for pseudo-differential operators when acting on Gelfand-Shilov spaces in [5] are straight-forward consequences of the results in Sect. 3. It is expected that the these results will also be useful in other situations. For example, these results are already applied in [2], where lifting properties between the modulation spaces above are established. We refer to $[19,24,25,30,31,33,36]$ and the references therein for more facts about pseudo-differential operators in framework of Gelfand-Shilov and modulation spaces.

Related questions were considered in the framework of the usual distribution theory in [32], where the pseudo-differential operators should have symbols in Hörmander classes of the form $S^{\left(\omega_{0}\right)}$, the set of all smooth $a$ which satisfies

$$
\left|\partial^{\alpha} a\right| \leq C_{\alpha} \omega_{0}
$$

More precisely, let $\mathscr{B}$ be a translation invariant BF-space, and that the condition (1.3) for $\omega$ and $\omega_{0}$ are replaced by the stronger estimate

$$
0<\omega(X+Y) \lesssim \omega(X)(1+|Y|)^{N}, \quad X, Y \in \mathbf{R}^{2 d}
$$

for some $N \geq 0$, and let $a \in S^{\left(\omega_{0}\right)}$. Then it is proved in [32, Theorem 3.2] that $\mathrm{Op}(a)$ is continuous from the modulation space $M\left(\omega_{0} \omega, \mathscr{B}\right)$ to $M(\omega, \mathscr{B})$. The obtained result in [32] can also be considered as extensions of certain results in the pioneering paper [29] by Tachizawa. For example, for suitable restrictions on $\omega, \omega_{0}$ and $\mathscr{B}$, it follows that [32, Theorem 3.2] covers [29, Theorem 2.1].

We observe the different conditions between on one hand the symbol classes $S^{\left(\omega_{0}\right)}$ in [32], and the other hand the classes $\Gamma_{S}^{\left(\omega_{0}\right)}$ and $\Gamma_{0, s}^{\left(\omega_{0}\right)}$ in Sect. 3. The symbols in $\Gamma_{S}^{\left(\omega_{0}\right)}$ and $\Gamma_{0, s}^{\left(\omega_{0}\right)}$ must obey Gevrey conditions of order $s$, while for the symbols in $S^{\left(\omega_{0}\right)}$, it is only required that they should be smooth. On the other hand, the weight $\omega_{0}$ in (1.4) in the definition of $S^{\left(\omega_{0}\right)}$ should satisfies (1.5) for some $N$, while for the definition of $\Gamma_{s}^{\left(\omega_{0}\right)}$ and $\Gamma_{0, s}^{\left(\omega_{0}\right)}$ this condition is relaxed into conditions of the form (1.5). This implies that the symbols in $\Gamma_{s}^{\left(\omega_{0}\right)}$ and $\Gamma_{0, s}^{\left(\omega_{0}\right)}$ are allowed to grow subexponentially, while the symbols in $S^{\left(\omega_{0}\right)}$ are not allowed to grow faster than polynomials. Consequently, more restrictions on growth are imposed on $S^{\left(\omega_{0}\right)}$ compared to $\Gamma_{s}^{\left(\omega_{0}\right)}$ and $\Gamma_{0, s}^{\left(\omega_{0}\right)}$.

In Sect. 4 we present some examples on continuity properties for pseudo-differential operators under considerations. These continuity properties are straight-forward consequences of the main results Theorems 3.5 and 3.8 in Sect. 3. Especially we explain continuity in the framework of Sobolev type spaces and weighted $L^{2}$ spaces, with exponential weights, as well as continuity of such operators on $\Gamma_{0, s}^{(\omega)}$ spaces. 


\section{Preliminaries}

In this section we discuss basic properties for modulation spaces and other related spaces. The proofs are in many cases omitted since they can be found in $[7-9,12-$ $15,17,34]$.

\subsection{Weight functions}

A weight or weight function on $\mathbf{R}^{d}$ is a positive function in $L_{l o c}^{\infty}\left(\mathbf{R}^{d}\right)$. Let $\omega$ and $v$ be weights on $\mathbf{R}^{d}$. Then $\omega$ is called $v$-moderate or moderate, if

$$
\omega\left(x_{1}+x_{2}\right) \lesssim \omega\left(x_{1}\right) v\left(x_{2}\right), \quad x_{1}, x_{2} \in \mathbf{R}^{d}
$$

Here $f(\theta) \lesssim g(\theta)$ means that $f(\theta) \leq c g(\theta)$ for some constant $c>0$ which is independent of $\theta$ in the domain of $f$ and $g$. If $v$ can be chosen as polynomial, then $\omega$ is called a weight of polynomial type. The weight function $v$ is called submultiplicative if it is even and (2.1) holds for $\omega=v$.

We let $\mathscr{P}_{E}\left(\mathbf{R}^{d}\right)$ be the set of all moderate weights on $\mathbf{R}^{d}$, and $\mathscr{P}\left(\mathbf{R}^{d}\right)$ be the subset of $\mathscr{P}_{E}\left(\mathbf{R}^{d}\right)$ which consists of all polynomially moderate functions on $\mathbf{R}^{d}$. We also let $\mathscr{P}_{E, s}\left(\mathbf{R}^{d}\right)\left(\mathscr{P}_{E, s}^{0}\left(\mathbf{R}^{d}\right)\right)$ be the set of all weights $\omega$ in $\mathbf{R}^{d}$ such that

$$
\omega\left(x_{1}+x_{2}\right) \lesssim \omega\left(x_{1}\right) e^{r\left|x_{2}\right|^{\frac{1}{s}}}, \quad x_{1}, x_{2} \in \mathbf{R}^{d}
$$

for some $r>0$ (for every $r>0$ ). We have

$$
\mathscr{P} \subseteq \mathscr{P}_{E, s_{1}}^{0} \subseteq \mathscr{P}_{E, s_{1}} \subseteq \mathscr{P}_{E, s_{2}}^{0} \subseteq \mathscr{P}_{E} \quad \text { when } s_{2}<s_{1}
$$

and

$$
\mathscr{P}_{E, s}=\mathscr{P}_{E} \quad \text { when } s \leq 1 \text {, }
$$

where the last equality follows from the fact that if $\omega \in \mathscr{P}_{E}\left(\mathbf{R}^{d}\right)\left(\omega \in \mathscr{P}_{E}^{0}\left(\mathbf{R}^{d}\right)\right)$, then

$$
\omega(x+y) \lesssim \omega(x) e^{r|y|} \text { and } e^{-r|x|} \leq \omega(x) \lesssim e^{r|x|}, \quad x, y \in \mathbf{R}^{d}
$$

hold true for some $r>0$ (for every $r>0$ ) (cf. [18]).

\subsection{Gelfand-Shilov spaces}

Let $0<h, s, \sigma \in \mathbf{R}$ be fixed. Then $\mathcal{S}_{s, h}^{\sigma}\left(\mathbf{R}^{d}\right)$ consists of all $f \in C^{\infty}\left(\mathbf{R}^{d}\right)$ such that

$$
\|f\|_{\mathcal{S}_{s, h}^{\sigma}} \equiv \sup \frac{\left|x^{\alpha} \partial^{\beta} f(x)\right|}{h^{|\alpha+\beta|} \alpha !^{s} \beta !^{\alpha}}
$$


is finite. Here the supremum should be taken over all $\alpha, \beta \in \mathbf{N}^{d}$ and $x \in \mathbf{R}^{d}$.

Obviously $\mathcal{S}_{s, h}^{\sigma}$ is a Banach space, contained in $\mathscr{S}$, and which increases with $h, s$ and $\sigma$ and $\mathcal{S}_{s, h}^{\sigma} \hookrightarrow \mathscr{S}$. Here and in what follows we use the notation $A \hookrightarrow B$ when the topological spaces $A$ and $B$ satisfy $A \subseteq B$ with continuous embeddings.

The Gelfand-Shilov spaces $\mathcal{S}_{s}^{\sigma}\left(\mathbf{R}^{d}\right)$ and $\Sigma_{s}^{\sigma}\left(\mathbf{R}^{d}\right)$ are defined as the inductive and projective limits respectively of $\mathcal{S}_{s, h}^{\sigma}\left(\mathbf{R}^{d}\right)$. This implies that

$$
\mathcal{S}_{s}^{\sigma}\left(\mathbf{R}^{d}\right)=\bigcup_{h>0} \mathcal{S}_{s, h}^{\sigma}\left(\mathbf{R}^{d}\right) \quad \text { and } \quad \Sigma_{s}^{\sigma}\left(\mathbf{R}^{d}\right)=\bigcap_{h>0} \mathcal{S}_{s, h}^{\sigma}\left(\mathbf{R}^{d}\right)
$$

and that the topology for $\mathcal{S}_{s}^{\sigma}\left(\mathbf{R}^{d}\right)$ is the strongest possible one such that the inclusion map from $\mathcal{S}_{s, h}^{\sigma}\left(\mathbf{R}^{d}\right)$ to $\mathcal{S}_{s}^{\sigma}\left(\mathbf{R}^{d}\right)$ is continuous, for every choice of $h>0$. The space $\Sigma_{s}^{\sigma}\left(\mathbf{R}^{d}\right)$ is a Fréchet space with seminorms $\|\cdot\|_{\mathcal{S}_{s, h}^{\sigma}}, h>0$. Moreover, $\Sigma_{s}^{\sigma}\left(\mathbf{R}^{d}\right) \neq\{0\}$, if and only if $s+\sigma \geq 1$ and $(s, \sigma) \neq\left(\frac{1}{2}, \frac{1}{2}\right)$, and $\mathcal{S}_{s}^{\sigma}\left(\mathbf{R}^{d}\right) \neq\{0\}$, if and only if $s+\sigma \geq 1$. If $s$ and $\sigma$ are chosen such that $\Sigma_{s}^{\sigma}\left(\mathbf{R}^{d}\right) \neq\{0\}$, then $\Sigma_{s}^{\sigma}\left(\mathbf{R}^{d}\right)$ is dense in $\mathscr{S}\left(\mathbf{R}^{d}\right)$ and in $\mathcal{S}_{s}^{\sigma}\left(\mathbf{R}^{d}\right)$. The same is true with $\mathcal{S}_{s}^{\sigma}\left(\mathbf{R}^{d}\right)$ in place of $\Sigma_{s}^{\sigma}\left(\mathbf{R}^{d}\right)$ (cf. [16]).

The Gelfand-Shilov distribution spaces $\left(\mathcal{S}_{s}^{\sigma}\right)^{\prime}\left(\mathbf{R}^{d}\right)$ and $\left(\Sigma_{s}^{\sigma}\right)^{\prime}\left(\mathbf{R}^{d}\right)$ are the projective and inductive limit respectively of $\left(\mathcal{S}_{s, h}^{\sigma}\right)^{\prime}\left(\mathbf{R}^{d}\right)$. This means that

$$
\left(\mathcal{S}_{s}^{\sigma}\right)^{\prime}\left(\mathbf{R}^{d}\right)=\bigcap_{h>0}\left(\mathcal{S}_{s, h}^{\sigma}\right)^{\prime}\left(\mathbf{R}^{d}\right) \text { and }\left(\Sigma_{s}^{\sigma}\right)^{\prime}\left(\mathbf{R}^{d}\right)=\bigcup_{h>0}\left(\mathcal{S}_{s, h}^{\sigma}\right)^{\prime}\left(\mathbf{R}^{d}\right)
$$

We remark that in [16] it is proved that $\left(\mathcal{S}_{s}^{\sigma}\right)^{\prime}\left(\mathbf{R}^{d}\right)$ is the dual of $\mathcal{S}_{s}^{\sigma}\left(\mathbf{R}^{d}\right)$, and $\left(\Sigma_{s}^{\sigma}\right)^{\prime}\left(\mathbf{R}^{d}\right)$ is the dual of $\Sigma_{s}^{\sigma}\left(\mathbf{R}^{d}\right)$ (also in topological sense). For conveniency we set

$$
\mathcal{S}_{s}=\mathcal{S}_{s}^{s}, \quad \mathcal{S}_{s}^{\prime}=\left(\mathcal{S}_{s}^{s}\right)^{\prime}, \quad \Sigma_{s}=\Sigma_{s}^{s} \quad \text { and } \quad \Sigma_{s}^{\prime}=\left(\Sigma_{s}^{s}\right)^{\prime}
$$

For every admissible $s, \sigma>0$ and $\varepsilon>0$ we have

$$
\begin{aligned}
\Sigma_{s}^{\sigma}\left(\mathbf{R}^{d}\right) & \hookrightarrow \mathcal{S}_{s}^{\sigma}\left(\mathbf{R}^{d}\right) \hookrightarrow \Sigma_{s+\varepsilon}^{\sigma+\varepsilon}\left(\mathbf{R}^{d}\right) \hookrightarrow \mathscr{S}\left(\mathbf{R}^{d}\right) \\
& \hookrightarrow \mathscr{S}^{\prime}\left(\mathbf{R}^{d}\right) \hookrightarrow\left(\Sigma_{s+\varepsilon}^{\sigma+\varepsilon}\right)^{\prime}\left(\mathbf{R}^{d}\right) \hookrightarrow\left(\mathcal{S}_{s}^{\sigma}\right)^{\prime}\left(\mathbf{R}^{d}\right) \hookrightarrow\left(\Sigma_{s}^{\sigma}\right)^{\prime}\left(\mathbf{R}^{d}\right)
\end{aligned}
$$

From now on we let $\mathscr{F}$ be the Fourier transform which takes the form

$$
(\mathscr{F} f)(\xi)=\widehat{f}(\xi) \equiv(2 \pi)^{-\frac{d}{2}} \int_{\mathbf{R}^{d}} f(x) e^{-i\langle x, \xi\rangle} d x
$$

when $f \in L^{1}\left(\mathbf{R}^{d}\right)$. Here $\langle\cdot, \cdot\rangle$ denotes the usual scalar product on $\mathbf{R}^{d}$. The map $\mathscr{F}$ extends uniquely to homeomorphisms on $\mathscr{S}^{\prime}\left(\mathbf{R}^{d}\right)$, from $\left(\mathcal{S}_{S}^{\sigma}\right)^{\prime}\left(\mathbf{R}^{d}\right)$ to $\left(\mathcal{S}_{\sigma}^{s}\right)^{\prime}\left(\mathbf{R}^{d}\right)$ and from $\left(\Sigma_{s}^{\sigma}\right)^{\prime}\left(\mathbf{R}^{d}\right)$ to $\left(\Sigma_{\sigma}^{s}\right)^{\prime}\left(\mathbf{R}^{d}\right)$. Furthermore, $\mathscr{F}$ restricts to homeomorphisms on $\mathscr{S}\left(\mathbf{R}^{d}\right)$, from $\mathcal{S}_{s}^{\sigma}\left(\mathbf{R}^{d}\right)$ to $\mathcal{S}_{\sigma}^{s}\left(\mathbf{R}^{d}\right)$ and from $\Sigma_{s}^{\sigma}\left(\mathbf{R}^{d}\right)$ to $\Sigma_{\sigma}^{s}\left(\mathbf{R}^{d}\right)$, and to a unitary operator on $L^{2}\left(\mathbf{R}^{d}\right)$. Similar facts hold true when $s=\sigma$ and the Fourier transform is replaced by a partial Fourier transform. 
Let $\phi \in \mathcal{S}_{S}^{\sigma}\left(\mathbf{R}^{d}\right)$ be fixed. Then the short-time Fourier transform $V_{\phi} f$ of $f \in$ $\left(\mathcal{S}_{s}^{\sigma}\right)^{\prime}\left(\mathbf{R}^{d}\right)$ with respect to the window function $\phi$ is the Gelfand-Shilov distribution on $\mathbf{R}^{2 d}$, defined by

$$
V_{\phi} f(x, \xi) \equiv\left(f, \phi(\cdot-x) e^{i\langle\cdot, \xi\rangle}\right)
$$

If in addition $f$ is an integrable function, then

$$
V_{\phi} f(x, \xi)=(2 \pi)^{-\frac{d}{2}} \int f(y) \overline{\phi(y-x)} e^{-i\langle y, \xi\rangle} d y
$$

Gelfand-Shilov spaces and their distribution spaces can in convenient ways be characterized by means of estimates of Fourier and short-time Fourier transforms (see e.g. [6,20,33,35]). Here some extension of the map $(f, \phi) \mapsto V_{\phi} f$ are also given, for example that this map is uniquely extendable to a continuous map from $\left(\mathcal{S}_{s}^{\sigma}\right)^{\prime}\left(\mathbf{R}^{d}\right) \times\left(\mathcal{S}_{S}^{\sigma}\right)^{\prime}\left(\mathbf{R}^{d}\right)$ to $\left(\mathcal{S}_{s, \sigma}^{\sigma, s}\right)^{\prime}\left(\mathbf{R}^{2 d}\right)$ (see also [1] for notations).

\subsection{Modulation spaces}

We recall that a quasi-norm $\|\cdot\|_{\mathscr{B}}$ of order $r \in(0,1]$ on the vector-space $\mathscr{B}$ over $\mathbf{C}$ is a nonnegative functional on $\mathscr{B}$ which satisfies

$$
\begin{aligned}
\|f+g\|_{\mathscr{B}} & \leq 2^{\frac{1}{r}-1}\left(\|f\|_{\mathscr{B}}+\|g\|_{\mathscr{B}}\right), \quad f, g \in \mathscr{B}, \\
\|\alpha \cdot f\|_{\mathscr{B}} & =|\alpha| \cdot\|f\|_{\mathscr{B}}, \quad \alpha \in \mathbf{C}, \quad f \in \mathscr{B}
\end{aligned}
$$

and

$$
\|f\|_{\mathscr{B}}=0 \quad \Leftrightarrow \quad f=0 .
$$

The vector space $\mathscr{B}$ is called a quasi-Banach space if it is a complete quasi-normed space. If $\mathscr{B}$ is a quasi-Banach space with quasi-norm satisfying (2.7) then on account of $[3,27]$ there is an equivalent quasi-norm to $\|\cdot\|_{\mathscr{B}}$ which additionally satisfies

$$
\|f+g\|_{\mathscr{B}}^{r} \leq\|f\|_{\mathscr{B}}^{r}+\|g\|_{\mathscr{B}}^{r}, \quad f, g \in \mathscr{B} .
$$

From now on we always assume that the quasi-norm of the quasi-Banach space $\mathscr{B}$ is chosen in such way that both (2.7) and (2.8) hold.

Let $\phi \in \Sigma_{1}\left(\mathbf{R}^{d}\right) \backslash 0, p, q \in(0, \infty]$ and $\omega \in \mathscr{P}_{E}\left(\mathbf{R}^{2 d}\right)$ be fixed. Then the modulation space $M_{(\omega)}^{p, q}\left(\mathbf{R}^{d}\right)$ consists of all $f \in \Sigma_{1}^{\prime}\left(\mathbf{R}^{d}\right)$ such that

$$
\|f\|_{M_{(\omega)}^{p, q}} \equiv\left(\int\left(\int\left|V_{\phi} f(x, \xi) \omega(x, \xi)\right|^{p} d x\right)^{q / p} d \xi\right)^{1 / q}<\infty
$$


(with the obvious modifications when $p=\infty$ and/or $q=\infty$ ). We set $M_{(\omega)}^{p}=M_{(\omega)}^{p, p}$, and if $\omega=1$, then we set $M^{p, q}=M_{(\omega)}^{p, q}$ and $M^{p}=M_{(\omega)}^{p}$.

The following proposition is a consequence of well-known facts in $[9,15,17,34]$. Here and in what follows, we let $p^{\prime}$ denotes the conjugate exponent of $p$, i.e.

$$
p^{\prime}= \begin{cases}\infty & \text { when } p \in(0,1] \\ \frac{p}{p-1} & \text { when } p \in(1, \infty) \\ 1 & \text { when } p=\infty .\end{cases}
$$

Proposition 2.1 Let $p, q, p_{j}, q_{j}, r \in(0, \infty]$ be such that $r \leq \min (1, p, q), j=1,2$, let $\omega, \omega_{1}, \omega_{2}, v \in \mathscr{P}_{E}\left(\mathbf{R}^{2 d}\right)$ be such that $\omega$ is $v$-moderate, $\phi \in M_{(v)}^{r}\left(\mathbf{R}^{d}\right) \backslash 0$, and let $f \in \Sigma_{1}^{\prime}\left(\mathbf{R}^{d}\right)$. Then the following is true:

(1) $f \in M_{(\omega)}^{p, q}\left(\mathbf{R}^{d}\right)$ if and only if (2.9) holds, i.e. $M_{(\omega)}^{p, q}\left(\mathbf{R}^{d}\right)$ is independent of the choice of $\phi$. Moreover, $M_{(\omega)}^{p, q}$ is a quasi-Banach space under the quasi-norm in (2.9), and different choices of $\phi$ give rise to equivalent quasi-norms.

If in addition $p, q \geq 1$, then $M_{(\omega)}^{p, q}\left(\mathbf{R}^{d}\right)$ is a Banach space with norm (2.9);

(2) if $p_{1} \leq p_{2}, q_{1} \leq q_{2}$ and $\omega_{2} \lesssim \omega_{1}$, then

$$
\Sigma_{1}\left(\mathbf{R}^{d}\right) \subseteq M_{\left(\omega_{1}\right)}^{p_{1}, q_{1}}\left(\mathbf{R}^{d}\right) \subseteq M_{\left(\omega_{2}\right)}^{p_{2}, q_{2}}\left(\mathbf{R}^{d}\right) \subseteq \Sigma_{1}^{\prime}\left(\mathbf{R}^{d}\right)
$$

We refer to $[9,12-15,17,28,34]$ for more facts about modulation spaces.

\subsection{A broader family of modulation spaces}

As announced in the introduction we consider in Sect. 3 mapping properties for pseudodifferential operators when acting on a broader class of modulation spaces, which are defined by imposing certain types of translation invariant solid BF-space norms on the short-time Fourier transforms. (Cf. [9-13].)

Definition 2.2 Let $\mathscr{B} \subseteq L_{l o c}^{r}\left(\mathbf{R}^{d}\right)$ be a quasi-Banach space of order $r \in(0,1]$, and let $v \in \mathscr{P}_{E}\left(\mathbf{R}^{d}\right)$. Then $\mathscr{B}$ is called a translation invariant Quasi-Banach function space on $\mathbf{R}^{d}$ (with respect to $v$ ), or invariant $Q B F$ space on $\mathbf{R}^{d}$, if there is a constant $C$ such that the following conditions are fulfilled:

(1) if $x \in \mathbf{R}^{d}$ and $f \in \mathscr{B}$, then $f(\cdot-x) \in \mathscr{B}$, and

$$
\|f(\cdot-x)\|_{\mathscr{B}} \leq C v(x)\|f\|_{\mathscr{B}}
$$

(2) if $f, g \in L_{l o c}^{r}\left(\mathbf{R}^{d}\right)$ satisfy $g \in \mathscr{B}$ and $|f| \leq|g|$, then $f \in \mathscr{B}$ and

$$
\|f\|_{\mathscr{B}} \leq C\|g\|_{\mathscr{B}} .
$$

If $v$ belongs to $\mathscr{P}_{E, s}\left(\mathbf{R}^{d}\right)\left(\mathscr{P}_{E, s}^{0}\left(\mathbf{R}^{d}\right)\right)$, then $\mathscr{B}$ in Definition 2.2 is called an invariant BF-space of Roumieu type (Beurling type) of order $s$. 

and

It follows from (2) in Definition 2.2 that if $f \in \mathscr{B}$ and $h \in L^{\infty}$, then $f \cdot h \in \mathscr{B}$,

$$
\|f \cdot h\|_{\mathscr{B}} \leq C\|f\|_{\mathscr{B}}\|h\|_{L^{\infty}} .
$$

If $r=1$, then $\mathscr{B}$ in Definition 2.2 is a Banach space, and the condition (2) means that a translation invariant QBF-space is a solid BF-space in the sense of (A.3) in [10]. The space $\mathscr{B}$ in Definition 2.2 is called an invariant $B F$-space (with respect to $v$ ) if $r=1$, and Minkowski's inequality holds true, i.e.

$$
\|f * \varphi\|_{\mathscr{B}} \lesssim\|f\|_{\mathscr{B}}\|\varphi\|_{L_{(v)}^{1}}, \quad f \in \mathscr{B}, \varphi \in C_{0}^{\infty}\left(\mathbf{R}^{d}\right)
$$

Example 2.3 Assume that $p, q \in[1, \infty]$, and let $L_{1}^{p, q}\left(\mathbf{R}^{2 d}\right)$ be the set of all $f \in$ $L_{l o c}^{1}\left(\mathbf{R}^{2 d}\right)$ such that

$$
\|f\|_{L_{1}^{p, q}} \equiv\left(\int\left(\int|f(x, \xi)|^{p} d x\right)^{q / p} d \xi\right)^{1 / q}
$$

if finite. Also let $L_{2}^{p, q}\left(\mathbf{R}^{2 d}\right)$ be the set of all $f \in L_{l o c}^{1}\left(\mathbf{R}^{2 d}\right)$ such that

$$
\|f\|_{L_{2}^{p, q}} \equiv\left(\int\left(\int|f(x, \xi)|^{q} d \xi\right)^{p / q} d x\right)^{1 / p}
$$

is finite. Then it follows that $L_{1}^{p, q}$ and $L_{2}^{p, q}$ are translation invariant BF-spaces with respect to $v=1$.

For translation invariant BF-spaces we make the following observation.

Proposition 2.4 Assume that $v \in \mathscr{P}_{E}\left(\mathbf{R}^{d}\right)$, and that $\mathscr{B}$ is an invariant BF-space with respect to $v$ such that (2.12) holds true. Then the convolution mapping $(\varphi, f) \mapsto \varphi * f$ from $C_{0}^{\infty}\left(\mathbf{R}^{d}\right) \times \mathscr{B}$ to $\mathscr{B}$ extends uniquely to a continuous mapping from $L_{(v)}^{1}\left(\mathbf{R}^{d}\right) \times \mathscr{B}$ to $\mathscr{B}$, and (2.12) holds true for any $f \in \mathscr{B}$ and $\varphi \in L_{(v)}^{1}\left(\mathbf{R}^{d}\right)$.

The result is a straight-forward consequence of the fact that $C_{0}^{\infty}$ is dense in $L_{(v)}^{1}$. Next we consider the extended class of modulation spaces which we are interested in.

Definition 2.5 Assume that $\mathscr{B}$ is a translation invariant QBF-space on $\mathbf{R}^{2 d}, \omega \in$ $\mathscr{P}_{E}\left(\mathbf{R}^{2 d}\right)$, and that $\phi \in \Sigma_{1}\left(\mathbf{R}^{d}\right) \backslash 0$. Then the set $M(\omega, \mathscr{B})$ consists of all $f \in \Sigma_{1}^{\prime}\left(\mathbf{R}^{d}\right)$ such that

$$
\|f\|_{M(\omega, \mathscr{B})} \equiv\left\|V_{\phi} f \omega\right\|_{\mathscr{B}}
$$

is finite. 
Obviously, we have $M_{(\omega)}^{p, q}\left(\mathbf{R}^{d}\right)=M(\omega, \mathscr{B})$ when $\mathscr{B}=L_{1}^{p, q}\left(\mathbf{R}^{2 d}\right)$ (cf. Example 2.3). It follows that many properties which are valid for the classical modulation spaces also hold for the spaces of the form $M(\omega, \mathscr{B})$. For example we have the following proposition, which shows that the definition of $M(\omega, \mathscr{B})$ is independent of the choice of $\phi$ when $\mathscr{B}$ is a Banach space. We omit the proof since it follows by similar arguments as in the proof of Proposition 11.3.2 in [17].

Proposition 2.6 Let $\mathscr{B}$ be an invariant BF-space with respect to $v_{0} \in \mathscr{P}_{E}\left(\mathbf{R}^{2 d}\right)$ for $j=1,2$. Also let $\omega, v \in \mathscr{P}_{E}\left(\mathbf{R}^{2 d}\right)$ be such that $\omega$ is $v$-moderate, $M(\omega, \mathscr{B})$ be the same as in Definition 2.5, and let $\phi \in M_{\left(v_{0} v\right)}^{1}\left(\mathbf{R}^{d}\right) \backslash 0$ and $f \in \Sigma_{1}^{\prime}\left(\mathbf{R}^{d}\right)$. Then $f \in M(\omega, \mathscr{B})$ if and only if $V_{\phi} f \omega \in \mathscr{B}$, and different choices of $\phi$ gives rise to equivalent norms in $M(\omega, \mathscr{B})$.

Next we recall the following result on completeness for $M(\omega, \mathscr{B})$. We refer to [35] for a proof of the first assertion and [22] for the second one.

Proposition 2.7 Let $\omega$ be a weight on $\mathbf{R}^{2 d}$, and let $\mathscr{B}$ be an invariant $Q B F$-space with respect to the submultiplicative $v \in \mathscr{P}_{E}\left(\mathbf{R}^{2 d}\right)$. Then the following is true:

(1) if in addition $\mathscr{B}$ is a mixed quasi-norm space of Lebesgue types, then $M(\omega, \mathscr{B})$ is a quasi-Banach space;

(2) if in addition $\mathscr{B}$ an invariant $B F$-space with respect to $v$, then $M(\omega, \mathscr{B})$ is a Banach space.

Finally we remark that certain modulation spaces without the condition on solidity are considered in [23].

\subsection{Mixed quasi-normed Lebesgue spaces}

In most cases, the quasi-Banach spaces $\mathscr{B}$ are mixed quasi-normed Lebesgue space, which are defined next. Let $E=\left\{e_{1}, \ldots, e_{d}\right\}$ be an orderd basis of $\mathbf{R}^{d}$. Then the corresponding lattice is

$$
\Lambda_{E}=\left\{j_{1} e_{1}+\cdots+j_{d} e_{d} ;\left(j_{1}, \ldots, j_{d}\right) \in \mathbf{Z}^{d}\right\}
$$

We define for each $\boldsymbol{q}=\left(q_{1}, \ldots, q_{d}\right) \in(0, \infty]^{d}$

$$
\max (\boldsymbol{q})=\max \left(q_{1}, \ldots, q_{d}\right) \text { and } \min (\boldsymbol{q})=\min \left(q_{1}, \ldots, q_{d}\right)
$$

Definition 2.8 Let $E=\left\{e_{1}, \ldots, e_{d}\right\}$ be an orderd basis of $\mathbf{R}^{d}, \omega$ be a weight on $\mathbf{R}^{d}$, $\boldsymbol{p}=\left(p_{1}, \ldots, p_{d}\right) \in(0, \infty]^{d}$ and $r=\min (1, \boldsymbol{p})$. If $f \in L_{l o c}^{r}\left(\mathbf{R}^{d}\right)$, then

$$
\|f\|_{L_{E,(\omega)}^{p}} \equiv\left\|g_{d-1}\right\|_{L^{p_{d}(\mathbf{R})}}
$$

where $g_{k}\left(z_{k}\right), z_{k} \in \mathbf{R}^{d-k}, k=0, \ldots, d-1$, are inductively defined as

$$
g_{0}\left(x_{1}, \ldots, x_{d}\right) \equiv\left|f\left(x_{1} e_{1}+\cdots+x_{d} e_{d}\right) \omega\left(x_{1} e_{1}+\cdots+x_{d} e_{d}\right)\right|
$$


and

$$
g_{k}\left(z_{k}\right) \equiv\left\|g_{k-1}\left(\cdot, z_{k}\right)\right\|_{L^{p_{k}(\mathbf{R})}, \quad k=1, \ldots, d-1 .}
$$

The space $L_{E,(\omega)}^{p}\left(\mathbf{R}^{d}\right)$ consists of all $f \in L_{l o c}^{r}\left(\mathbf{R}^{d}\right)$ such that $\|f\|_{L_{E,(\omega)}^{p}}$ is finite, and is called E-split Lebesgue space (with respect to $\boldsymbol{p}$ and $\omega$ ).

Let $E, \boldsymbol{p}$ and $\omega$ be the same as in Definition 2.8. Then the discrete version $\ell_{E,(\omega)}^{\boldsymbol{p}}\left(\Lambda_{E}\right)$ of $L_{E,(\omega)}^{p}\left(\mathbf{R}^{d}\right)$ is the set of all sequences $a=\{a(j)\}_{j \in \Lambda_{E}}$ such that the quasi-norm

$$
\|a\|_{\ell_{E,(\omega)}^{p}} \equiv\left\|f_{a}\right\|_{L_{E,(\omega)}^{p}}, \quad f_{a}=\sum_{j \in \Lambda_{E}} a(j) \chi_{j}
$$

is finite. Here $\chi_{j}$ is the characteristic function of $j+\kappa(E)$, where $\kappa(E)$ is the parallelepiped spanned by the basis $E$. We also set $L_{E}^{\boldsymbol{p}}=L_{E,(\omega)}^{\boldsymbol{p}}$ and $\ell_{E}^{\boldsymbol{p}}=\ell_{E,(\omega)}^{\boldsymbol{p}}$ when $\omega=1$.

Definition 2.9 Let $E$ be an ordered basis of the phase space $\mathbf{R}^{2 d}$. Then $E$ is called phase split if there is a subset $E_{0} \subseteq E$ such that the span of $E_{0}$ equals $\{(x, 0) \in$ $\left.\mathbf{R}^{2 d} ; x \in \mathbf{R}^{d}\right\}$, and the span of $E_{0}$ and $E \backslash E_{0}$ equals $\left\{(x, 0) \in \mathbf{R}^{2 d} ; \xi \in \mathbf{R}^{d}\right\}$ respective $\left\{(0, \xi) \in \mathbf{R}^{2 d} ; \xi \in \mathbf{R}^{d}\right\}$.

\subsection{Pseudo-differential operators}

Next we recall some facts on pseudo-differential operators. For any set $\Omega, \mathrm{M}(\mathrm{d}, \Omega)$ is the set of all $d \times d$-matrices with entries in $\Omega$. Let $A \in \mathbf{M}(d, \mathbf{R})$ be fixed and let $a \in \Sigma_{1}\left(\mathbf{R}^{2 d}\right)$. Then the pseudo-differential operator $\mathrm{Op}_{A}(a)$ is the linear and continuous operator on $\Sigma_{1}\left(\mathbf{R}^{d}\right)$, defined by the formula

$$
\begin{aligned}
& \left(\mathrm{Op}_{A}(a) f\right)(x) \\
& =(2 \pi)^{-d} \iint a(x-A(x-y), \xi) f(y) e^{i\langle x-y, \xi\rangle} d y d \xi
\end{aligned}
$$

The definition of $\mathrm{Op}_{A}(a)$ extends to any $a \in \Sigma_{1}^{\prime}\left(\mathbf{R}^{2 d}\right)$, and then $\mathrm{Op}_{A}(a)$ is continuous from $\Sigma_{1}\left(\mathbf{R}^{d}\right)$ to $\Sigma_{1}^{\prime}\left(\mathbf{R}^{d}\right)$. Moreover, for every fixed $A \in \mathbf{M}(d, \mathbf{R})$, it follows that there is a one to one correspondence between such operators, and pseudo-differential operators of the form $\mathrm{Op}_{A}(a)$. (See e.g. [21].) If $A=2^{-1} I$, where $I \in \mathbf{M}(d, \mathbf{R})$ is the identity matrix, then $\mathrm{Op}_{A}(a)$ is equal to the Weyl operator $\mathrm{Op}^{w}(a)$ of $a$. If instead $A=0$, then the standard (Kohn-Nirenberg) representation $\operatorname{Op}(a)=a(x, D)$ is obtained.

If $a_{1}, a_{2} \in \Sigma_{1}^{\prime}\left(\mathbf{R}^{2 d}\right)$ and $A_{1}, A_{2} \in \mathbf{M}(d, \mathbf{R})$, then

$$
\mathrm{Op}_{A_{1}}\left(a_{1}\right)=\mathrm{Op}_{A_{2}}\left(a_{2}\right) \Leftrightarrow a_{2}(x, \xi)=e^{i\left\langle\left(A_{1}-A_{2}\right) D_{\xi}, D_{x}\right\rangle} a_{1}(x, \xi)
$$

(Cf. [21].) 
The following special case of [36, Theorem 3.1] is important when discussing continuity of pseudo-differential operators when acting on quasi-Banach modulation spaces.

Proposition 2.10 Let $\omega_{1}, \omega_{2} \in \mathscr{P}_{E}\left(\mathbf{R}^{2 d}\right)$ and $\omega_{0} \in \mathscr{P}_{E}\left(\mathbf{R}^{2 d} \oplus \mathbf{R}^{2 d}\right)$ be such that

$$
\frac{\omega_{2}(x, \xi)}{\omega_{1}(y, \eta)} \lesssim \omega_{0}(x, \eta, \xi-\eta, y-x)
$$

Also let $\boldsymbol{p} \in(0, \infty]^{2 d}$, E be a phase split basis of $\mathbf{R}^{2 d}$ and let $a \in M_{\left(\omega_{0}\right)}^{\infty, 1}\left(\mathbf{R}^{2 d}\right)$. Then $\mathrm{Op}_{0}(a)$ is continuous from $M\left(L^{p, E}, \omega_{1}\right)$ to $M\left(L^{p, E}, \omega_{2}\right)$.

In the next section we discuss continuity for pseudo-differential operators with symbols in the following definition. (See also the Sect. 1.)

Definition 2.11 Let $\omega_{0}$ be a weight on $\mathbf{R}^{d}$, and let $s \geq 0$.

(1) The set $\Gamma_{s}^{\left(\omega_{0}\right)}\left(\mathbf{R}^{d}\right)$ consists of all $a \in C^{\infty}\left(\mathbf{R}^{d}\right)$ such that

$$
\left|\partial^{\alpha} f(x)\right| \lesssim h^{|\alpha|} \alpha !^{s} \omega_{0}(x), \quad \alpha \in \mathbf{N}^{d}
$$

for some constant $h>0$;

(2) The set $\Gamma_{0, s}^{\left(\omega_{0}\right)}\left(\mathbf{R}^{d}\right)$ consists of all $a \in C^{\infty}\left(\mathbf{R}^{d}\right)$ such that (2.16) holds for every constant $h>0$.

Remark 2.12 We have

$$
\mathscr{P} \subseteq \mathscr{P}_{E, s_{1}}^{0} \subseteq \mathscr{P}_{E, s_{1}} \subseteq \mathscr{P}_{E, s_{2}}^{0}, \quad s_{2}<s_{1}
$$

Hence, despite that $\Gamma_{0, s}^{\left(\omega_{0}\right)} \subseteq \Gamma_{s}^{\left(\omega_{0}\right)} \subseteq S^{\left(\omega_{0}\right)}$ holds for every $\omega_{0}$, we have

$$
\Gamma_{0, s}^{(\omega)} \nsubseteq \bigcup_{\omega_{0} \in \mathscr{P}} S^{\left(\omega_{0}\right)}
$$

for some $\omega \in \mathscr{P}_{E, s}^{0}$, and

$$
\Gamma_{0, s}^{(\omega)} \nsubseteq \bigcup_{\omega_{0} \in \mathscr{P}_{E, s}^{0}} \Gamma_{s}^{\left(\omega_{0}\right)}
$$

for some $\omega \in \mathscr{P}_{E, s}$.

\section{Continuity for pseudo-differential operators with symbols in $\Gamma_{s}^{(\omega)}$ and $\Gamma_{0,5}^{(\omega)}$}

In this section we discuss continuity for operators in $\mathrm{Op}\left(\Gamma_{s}^{\left(\omega_{0}\right)}\right)$ and $\mathrm{Op}\left(\Gamma_{0, s}^{\left(\omega_{0}\right)}\right)$ when acting on a general class of modulation spaces. In Theorem 3.5 below it is proved 
that if $\omega, \omega_{0} \in \mathscr{P}_{E, s}^{0}, A \in \mathbf{M}(d, \mathbf{R})$ and $a \in \Gamma_{0, s}^{\left(\omega_{0}\right)}$, then $\mathrm{Op}_{A}(a)$ is continuous from $M\left(\omega_{0} \omega, \mathscr{B}\right)$ to $M\left(\omega_{0}, \mathscr{B}\right)$. This gives an analogy to [32, Theorem 3.2] in the framework of operator theory and Gelfand-Shilov classes.

We need some preparations before discussing these mapping properties. The following result shows that for any weight in $\mathscr{P}_{E}$, there are equivalent weights that satisfy strong Gevrey regularity.

Proposition 3.1 Let $\omega \in \mathscr{P}_{E}\left(\mathbf{R}^{d}\right)$ and $s>0$. Then there exists a weight $\omega_{0} \in$ $\mathscr{P}_{E}\left(\mathbf{R}^{d}\right) \cap C^{\infty}\left(\mathbf{R}^{d}\right)$ such that the following is true:

(1) $\omega_{0} \asymp \omega$;

(2) $\left|\partial^{\alpha} \omega_{0}(x)\right| \lesssim \omega_{0}(x) h^{|\alpha|} \alpha !^{s} \asymp \omega(x) h^{|\alpha|} \alpha !^{s}$ for every $h>0$.

Proof We may assume that $s<\frac{1}{2}$. It suffices to prove that (2) should hold for some $h>0$. Let $\phi_{0} \in \Sigma_{1-s}^{s}\left(\mathbf{R}^{d}\right) \backslash 0$, and let $\phi=\left|\phi_{0}\right|^{2}$. Then $\phi \in \Sigma_{1-s}^{s}\left(\mathbf{R}^{d}\right)$ is non-negative. In particular,

$$
\left|\partial^{\alpha} \phi(x)\right| \lesssim h^{|\alpha|} \alpha !^{s} e^{-c|x|^{\frac{1}{1-s}}}
$$

for every $h>0$ and $c>0$. We set $\omega_{0}=\omega * \phi$.

Then

$$
\begin{aligned}
\left|\partial^{\alpha} \omega_{0}(x)\right| & =\left|\int \omega(y)\left(\partial^{\alpha} \phi\right)(x-y) d y\right| \\
& \lesssim h^{|\alpha|} \alpha !^{s} \int \omega(y) e^{-c|x-y|^{\frac{1}{1-s}}} d y \\
& \lesssim h^{|\alpha|} \alpha !^{s} \int \omega(x+(y-x)) e^{-c|x-y|^{\frac{1}{1-s}}} d y \\
& \lesssim h^{|\alpha|} \alpha !^{s} \omega(x) \int e^{-\frac{c}{2}|x-y|^{\frac{1}{1-s}}} d y \asymp h^{|\alpha|} \alpha !^{s} \omega(x),
\end{aligned}
$$

where the last inequality follows (2.2) and the fact that $\phi$ is bounded by a super exponential function. This gives the first part of (2).

The equivalences in (1) follows in the same way as in e.g. [33]. More precisely, by (2.2) we have

$$
\begin{aligned}
\omega_{0}(x) & =\int \omega(y) \phi(x-y) d y=\int \omega(x+(y-x)) \phi(x-y) d y \\
& \lesssim \omega(x) \int e^{c|x-y|} \phi(x-y) d y \asymp \omega(x) .
\end{aligned}
$$

In the same way, (2.3) gives 


$$
\begin{aligned}
\omega_{0}(x) & =\int \omega(y) \phi(x-y) d y=\int \omega(x+(y-x)) \phi(x-y) d y \\
& \gtrsim \omega(x) \int e^{-c|x-y|} \phi(x-y) d y \asymp \omega(x),
\end{aligned}
$$

and (1) as well as the second part of (2) follow.

The next result shows that $\Gamma_{s}^{(\omega)}$ and $\Gamma_{0, s}^{(\omega)}$ can be characterised in terms of estimates of short-time Fourier transforms.

Proposition 3.2 Let $s \geq 1, \phi \in \mathcal{S}_{S}\left(\mathbf{R}^{d}\right) \backslash 0$, and let $f \in \mathcal{S}_{1 / 2}^{\prime}\left(\mathbf{R}^{d}\right)$. Then the following is true:

(1) If $\omega \in \mathscr{P}_{E, s}^{0}\left(\mathbf{R}^{d}\right)$, then $f \in C^{\infty}\left(\mathbf{R}^{d}\right)$ and satisfies

$$
\left|\partial^{\alpha} f(x)\right| \lesssim \omega(x) h^{|\alpha|} \alpha !^{s}
$$

for some $h>0$, if and only if

$$
\left|V_{\phi} f(x, \xi)\right| \lesssim \omega(x) e^{-r|\xi|^{\frac{1}{s}}}
$$

for some $r>0$;

(2) If $\omega \in \mathscr{P}_{E, s}\left(\mathbf{R}^{d}\right)$ and in addition $\phi \in \Sigma_{s}\left(\mathbf{R}^{d}\right)$, then $f \in C^{\infty}\left(\mathbf{R}^{d}\right)$ and satisfies (3.1) for every $h>0$ (resp. for some $h>0$ ), if and only if (3.2) holds true for every $r>0$ (resp. for some $r>0$ ).

Proof We shall follow the proof of Proposition 3.1 in [5]. We only prove (2), and then when (3.1) or (3.2) are true for every $\varepsilon>0$. The other cases follow by similar arguments and are left for the reader.

Assume that $\phi \in \Sigma_{S}\left(\mathbf{R}^{d}\right), \omega \in \mathscr{P}_{E, s}\left(\mathbf{R}^{d}\right)$ and that (3.1) holds for every $\varepsilon>0$. Then for every $x \in \mathbf{R}^{d}$ the function

$$
y \mapsto F_{x}(y) \equiv f(y+x) \overline{\phi(y)}
$$

belongs to $\Sigma_{s}$, and $\omega(x+y) \lesssim e^{h_{0}|y|^{\frac{1}{S}}} \omega(x)$ for some $h_{0}>0$. By a straight-forward application of Leibnitz formula and the facts that

$$
\left|\partial^{\alpha} \phi(x)\right| \lesssim \varepsilon^{|\alpha|} \alpha !^{s} e^{-h|x|^{\frac{1}{s}}} \text { and } \omega(x+y) \lesssim \omega(x) e^{h_{0}|y|^{\frac{1}{s}}}
$$

for some $h_{0}>0$ and every $\varepsilon, h>0$ we get

$$
\left|\partial_{y}^{\alpha} F_{x}(y)\right| \lesssim \omega(x) e^{-h|y|^{\frac{1}{s}}} \varepsilon^{|\alpha|} \alpha !^{s}
$$

for every $\varepsilon, h>0$. In particular,

$$
\left|F_{x}(y)\right| \lesssim \omega(x) e^{-h|y|^{\frac{1}{s}}} \text { and }\left|\widehat{F}_{x}(\xi)\right| \lesssim \omega(x) e^{-h|\xi|^{\frac{1}{s}}}
$$


for every $h>0$. Since $\left|V_{\phi} f(x, \xi)\right|=\left|\widehat{F}_{x}(\xi)\right|$, the estimate (3.2) follows from the second inequality in (3.3). This shows that if (3.1) holds for every $\varepsilon>0$, then (3.2) holds for every $\varepsilon>0$.

Next suppose that (3.2) holds for every $\varepsilon>0$. By Fourier's inversion formula we get

$$
f(x)=(2 \pi)^{-\frac{d}{2}}\|\phi\|_{L^{2}}^{-2} \iint_{\mathbf{R}^{2 d}} V_{\phi} f(y, \eta) \phi(x-y) e^{i\langle x, \eta\rangle} d y d \eta
$$

By differentiation and the fact that $\phi \in \Sigma_{S}$ we get

$$
\begin{aligned}
\left|\partial^{\alpha} f(x)\right| & \asymp\left|\sum_{\beta \leq \alpha}\left(\begin{array}{l}
\alpha \\
\beta
\end{array}\right) i^{|\beta|} \iint_{\mathbf{R}^{2 d}} \eta^{\beta} V_{\phi} f(y, \eta)\left(\partial^{\alpha-\beta} \phi\right)(x-y) e^{i\langle x, \eta\rangle} d y d \eta\right| \\
& \leq \sum_{\beta \leq \alpha}\left(\begin{array}{l}
\alpha \\
\beta
\end{array}\right) \iint_{\mathbf{R}^{2 d}}\left|\eta^{\beta} V_{\phi} f(y, \eta)\left(\partial^{\alpha-\beta} \phi\right)(x-y)\right| d y d \eta \\
& \lesssim \sum_{\beta \leq \alpha}\left(\begin{array}{l}
\alpha \\
\beta
\end{array}\right) \iint_{\mathbf{R}^{2 d}}\left|\eta^{\beta} \omega(y) e^{-\varepsilon_{3}|\eta|^{\frac{1}{s}}}\left(\partial^{\alpha-\beta} \phi\right)(x-y)\right| d y d \eta \\
& \lesssim \sum_{\beta \leq \alpha}\left(\begin{array}{l}
\alpha \\
\beta
\end{array}\right) \varepsilon_{2}^{|\alpha-\beta|}(\alpha-\beta) !^{s} \iint_{\mathbf{R}^{2 d}}\left|\eta^{\beta}\right| \omega(y) e^{-\varepsilon_{3}|\eta|^{\frac{1}{s}}} e^{-\varepsilon_{1}|x-y|^{\frac{1}{s}}} d y d \eta
\end{aligned}
$$

for every $\varepsilon_{1}, \varepsilon_{2}, \varepsilon_{3}>0$. Since

$$
\left|\eta^{\beta} e^{-\varepsilon_{3}|\eta|^{\frac{1}{s}}}\right| \lesssim \varepsilon_{2}^{|\beta|} \beta !^{s} e^{-\varepsilon_{3}|\eta|^{\frac{1}{s}} / 2}
$$

when $\varepsilon_{3}$ is chosen large enough compared to $\varepsilon_{2}^{-1}$, we get

$$
\begin{aligned}
\left|\partial^{\alpha} f(x)\right| & \lesssim \varepsilon_{2}^{|\alpha|} \sum_{\beta \leq \alpha}\left(\begin{array}{c}
\alpha \\
\beta
\end{array}\right)(\beta !(\alpha-\beta) !)^{s} \iint_{\mathbf{R}^{2 d}} \omega(y) e^{-\varepsilon_{3}|\eta|^{\frac{1}{s}} / 2} e^{-\varepsilon_{1}|x-y|^{\frac{1}{s}}} d y d \eta \\
& \lesssim\left(2 \varepsilon_{2}\right)^{|\alpha|} \alpha !^{s} \int_{\mathbf{R}^{n}} \omega(y) e^{-\varepsilon_{1}|x-y|^{\frac{1}{s}}} d y
\end{aligned}
$$

Since $\omega(y) \leq \omega(x) e^{h_{0}|x-y|^{\frac{1}{s}}}$ for some $h_{0} \geq 0$ and $\varepsilon_{1}$ can be chosen arbitrarily large, it follows from the last estimate that

$$
\left|\partial^{\alpha} f(x)\right| \lesssim\left(2 \varepsilon_{2}\right)^{|\alpha|} \alpha !^{s} \omega(x)
$$

for every $\varepsilon_{2}>0$, and the result follows.

The following result is now a straight-forward consequence of the previous proposition and the definitions. 
Proposition 3.3 Let $s \geq 1, q \in(0, \infty], \omega_{0} \in \mathscr{P}_{E, s}\left(\mathbf{R}^{d}\right)$ and let $\omega_{r}(x, \xi)=$ $\omega_{0}(x) e^{-r|\xi|^{\frac{1}{s}}}$ when $x, \xi \in \mathbf{R}^{d}$. Then

$$
\bigcup_{r>0} M_{\left(1 / \omega_{r}\right)}^{\infty, q}\left(\mathbf{R}^{d}\right)=\Gamma_{s}^{\left(\omega_{0}\right)}\left(\mathbf{R}^{d}\right) \text { and } \bigcap_{r>0} M_{\left(1 / \omega_{r}\right)}^{\infty, q}\left(\mathbf{R}^{d}\right)=\Gamma_{0, s}^{\left(\omega_{0}\right)}\left(\mathbf{R}^{d}\right)
$$

The following lemma is a consequence of Theorem 4.6 in [5].

Lemma 3.4 Let $s \geq 1 \omega \in \mathscr{P}_{E}\left(\mathbf{R}^{2 d}\right), A_{1}, A_{2} \in \mathbf{M}(d, \mathbf{R})$, and that $a_{1}, a_{2} \in \Sigma_{1}^{\prime}\left(\mathbf{R}^{2 d}\right)$ are such that $\mathrm{Op}_{A_{1}}\left(a_{1}\right)=\mathrm{Op}_{A_{2}}\left(a_{2}\right)$. Then

$$
a_{1} \in \Gamma_{s}^{(\omega)}\left(\mathbf{R}^{2 d}\right) \quad \Leftrightarrow \quad a_{2} \in \Gamma_{s}^{(\omega)}\left(\mathbf{R}^{2 d}\right)
$$

and

$$
a_{1} \in \Gamma_{0, s}^{(\omega)}\left(\mathbf{R}^{2 d}\right) \quad \Leftrightarrow \quad a_{2} \in \Gamma_{0, s}^{(\omega)}\left(\mathbf{R}^{2 d}\right)
$$

We have now the following result.

Theorem 3.5 Let $A \in \mathbf{M}(d, \mathbf{R}), s \geq 1, \omega, \omega_{0} \in \mathscr{P}_{E, s}^{0}\left(\mathbf{R}^{2 d}\right), a \in \Gamma_{s}^{\left(\omega_{0}\right)}\left(\mathbf{R}^{2 d}\right)$, and that $\mathscr{B}$ is an invariant $B F$-space on $\mathbf{R}^{2 d}$. Then $\mathrm{Op}_{A}\left(\right.$ a) is continuous from $M\left(\omega_{0} \omega, \mathscr{B}\right)$ to $M(\omega, \mathscr{B})$.

We need some preparations for the proof, and start with the following lemma.

Lemma 3.6 Suppose $s \geq 1, \omega \in \mathscr{P}_{E}\left(\mathbf{R}^{d_{0}}\right)$ and that $f \in C^{\infty}\left(\mathbf{R}^{d+d_{0}}\right)$ satisfies

$$
\left|\partial^{\alpha} f(x, y)\right| \lesssim h^{|\alpha|} \alpha !^{s} e^{-r|x|^{\frac{1}{s}}} \omega(y), \quad \alpha \in \mathbf{N}^{d+d_{0}}
$$

for some $h>0$ and $r>0$. Then there are $f_{0} \in C^{\infty}\left(\mathbf{R}^{d+d_{0}}\right)$ and $\psi \in \mathcal{S}_{S}\left(\mathbf{R}^{d}\right)$ such that (3.4) holds with $f_{0}$ in place of $f$ for some $h>0$ and $r>0$, and $f(x, y)=$ $f_{0}(x, y) \psi(x)$.

Proof By Proposition 3.1 there is a submultiplicative weight $v_{0} \in \mathscr{P}_{E, s}\left(\mathbf{R}^{d}\right) \cap$ $C^{\infty}\left(\mathbf{R}^{d}\right)$ such that

$$
v_{0}(x) \asymp e^{\frac{r}{2}|x|^{\frac{1}{s}}}
$$

and

$$
\left|\partial^{\alpha} v_{0}(x)\right| \lesssim h^{|\alpha|} \alpha !^{s} v_{0}(x), \quad \alpha \in \mathbf{N}^{d}
$$

for some $h>0$. Since $s \geq 1$, a straight-forward combination of Faà di Bruno's formula and (3.6) gives 


$$
\left|\partial^{\alpha}\left(\frac{1}{v_{0}(x)}\right)\right| \lesssim h^{|\alpha|} \alpha !^{s} \cdot \frac{1}{v_{0}(x)}, \quad \alpha \in \mathbf{N}^{d}
$$

for some $h>0$. It follows from (3.5) and (3.6) that if $\psi=1 / v$, then $\psi \in \mathcal{S}_{S}\left(\mathbf{R}^{d}\right)$. Furthermore, if $f_{0}(x, y)=f(x, y) v_{0}(x)$, then an application of Leibnitz formula gives

$$
\begin{aligned}
& \left|\partial_{x}^{\alpha} \partial_{y}^{\alpha_{0}} f_{0}(x, y)\right| \lesssim \sum_{\gamma \leq \alpha}\left(\begin{array}{l}
\alpha \\
\gamma
\end{array}\right)\left|\partial_{x}^{\delta_{x}} \partial_{y}^{\alpha_{0}} f(x, y)\right|\left|\partial^{\alpha-\delta} v_{0}(x)\right| \\
& \lesssim h^{|\alpha|+\left|\alpha_{0}\right|} \sum_{\gamma \leq \alpha}\left(\begin{array}{l}
\alpha \\
\gamma
\end{array}\right)\left(\gamma ! \alpha_{0} !\right)^{s} e^{-r|x|^{\frac{1}{s}}} \omega(y)(\alpha-\gamma) !^{s} v_{0}(x) \\
& \lesssim(2 h)^{|\alpha|+\left|\alpha_{0}\right|}\left(\alpha ! \alpha_{0} !\right)^{s} e^{-r|x|^{\frac{1}{s}}} v_{0}(x) \omega(y) \\
& \asymp(2 h)^{|\alpha|+\left|\alpha_{0}\right|}\left(\alpha ! \alpha_{0} !\right)^{s} e^{-\frac{r}{2}|x|^{\frac{1}{s}}} \omega(y)
\end{aligned}
$$

for some $h>0$, which gives the desired estimate on $f_{0}$, The result now follows since it is evident that $f(x, y)=f_{0}(x, y) \psi(x)$.

For the next lemma we recall that for any $a \in \Sigma_{1}^{\prime}\left(\mathbf{R}^{2 d}\right)$ there is a unique $b \in$ $\Sigma_{1}^{\prime}\left(\mathbf{R}^{2 d}\right)$ such that $\mathrm{Op}(a)^{*}=\mathrm{Op}(b)$, and then $b(x, \xi)=e^{i\left\langle D_{\xi}, D_{x}\right\rangle} \overline{a(x, \xi)}$ in view of [21, Theorem 18.1.7]. Furthermore, by the latter equality and [5, Theorem 4.1] it follows that

$$
a \in \Gamma_{s}^{(\omega)}\left(\mathbf{R}^{2 d}\right) \Leftrightarrow b \in \Gamma_{s}^{(\omega)}\left(\mathbf{R}^{2 d}\right) .
$$

Lemma 3.7 Let $s \geq 1, \omega \in \mathscr{P}_{E, s}^{0}\left(\mathbf{R}^{2 d}\right), \vartheta \in \mathscr{P}_{E, s}^{0}\left(\mathbf{R}^{d}\right)$ and $v \in \mathscr{P}_{E, s}^{0}\left(\mathbf{R}^{d}\right)$ be such that $v$ is submultiplicative, $\omega \in \Gamma_{0, s}^{(\omega)}\left(\mathbf{R}^{2 d}\right)$ is $v \otimes v$-moderate, $\vartheta=v^{-\frac{1}{2}}$ and $\vartheta \in$ $\Gamma_{0, s}^{(\vartheta)}\left(\mathbf{R}^{d}\right)$. Also let $a \in \Gamma_{s}^{(\omega)}\left(\mathbf{R}^{2 d}\right)$, choose $b \in \Gamma_{s}^{(\omega)}\left(\mathbf{R}^{2 d}\right)$ such that $\operatorname{Op}(b)=\operatorname{Op}(a)^{*}$, $f \in \mathcal{S}_{S}\left(\mathbf{R}^{d}\right), \phi \in \Sigma_{s}\left(\mathbf{R}^{d}\right), \varphi=\widehat{\phi} \cdot v$,

$$
\Phi(x, \xi, y, \eta)=\frac{b(y, \xi+\eta)}{\omega(x, \xi) v(x-y) v(\eta)}
$$

and

$$
H(x, \xi, y)=v(x-y) \int \Phi(x, \xi, y, \eta) \varphi(\eta) e^{i\langle y-x, \eta\rangle} d \eta
$$

Then

$$
V_{\phi}(\mathrm{Op}(a) f)(x, \xi)=(2 \pi)^{-d}\left(f, e^{i\langle\cdot, \xi\rangle} H(x, \xi, \cdot)\right) \omega(x, \xi)
$$

Furthermore the following is true: 
(1) $H \in C^{\infty}\left(\mathbf{R}^{3 d}\right)$ and satisfies

$$
\left|\partial^{\alpha} H(x, \xi, y)\right| \lesssim h_{0}^{|\alpha|} \alpha !^{s} e^{-r_{0}|x-y|^{\frac{1}{s}}},
$$

for some $h_{0}, r_{0}>0$;

(2) there are functions $H_{0} \in C^{\infty}\left(\mathbf{R}^{3 d}\right)$ and $\phi_{0} \in \mathcal{S}_{S}\left(\mathbf{R}^{d}\right)$ such that

$$
H(x, \xi, y)=H_{0}(x, \xi, y) \phi_{0}(y-x),
$$

and such that (3.9) holds for some $h_{0}, r_{0}>0$, with $H_{0}$ in place of $H$.

Proof When proving the first part, we shall use some ideas in the proof of [32, Lemma 3.3]. By straight-forward computations we get

$$
\begin{aligned}
V_{\phi}(\mathrm{Op}(a) f)(x, \xi) & =(2 \pi)^{-\frac{d}{2}}\left(\mathrm{Op}(a) f, \phi(\cdot-x) e^{i\langle\cdot, \xi\rangle}\right) \\
& =(2 \pi)^{-\frac{d}{2}}\left(f, \mathrm{Op}(b)\left(\phi(\cdot-x) e^{i\langle\cdot, \xi\rangle}\right)\right) \\
& =(2 \pi)^{-d}\left(f, e^{i\langle\cdot, \xi\rangle} H_{1}(x, \xi, \cdot)\right) \omega(x, \xi),
\end{aligned}
$$

where

$$
\begin{aligned}
H_{1}(x, \xi, y) & =(2 \pi)^{\frac{d}{2}} e^{-i\langle y, \xi\rangle}\left(\operatorname{Op}(b)\left(\phi(\cdot-x) e^{i\langle\cdot, \xi\rangle}\right)\right)(y) / \omega(x, \xi) \\
& =\int \frac{b(y, \eta)}{\omega(x, \xi)} \widehat{\phi}(\eta-\xi) e^{-i\langle x-y, \eta-\xi\rangle} d \eta \\
& =v(x-y) \int \Phi(x, \xi, y, \eta-\xi) \varphi(\eta-\xi) e^{-i\langle x-y, \eta-\xi\rangle} d \eta
\end{aligned}
$$

If $\eta-\xi$ are taken as new variables of integrations, it follows that the right-hand side is equal to $H(x, y, \xi)$. This gives the first part of the lemma.

In order to prove (1), let

$$
\Phi_{0}(x, \xi, y, \eta)=\Phi(x, \xi, y, \eta) \varphi(\eta)
$$

and let $\Psi=\mathscr{F}_{4} \Phi_{0}$, where $\mathscr{F}_{4} \Phi_{0}$ is the partial Fourier transform of $\Phi_{0}(x, \xi, y, \eta)$ with respect to the $\eta$ variable. Then it follows from the assumptions that

$$
\left|\partial^{\alpha} \Phi_{0}(x, \xi, y, \eta)\right| \lesssim h_{0}^{|\alpha|} \alpha !^{s} e^{-r_{0}|\eta|^{\frac{1}{s}}}
$$

for some $h_{0}, r_{0}>0$, which shows that $\eta \mapsto \Phi_{0}(x, \xi, y, \eta)$ is an element in $\mathcal{S}_{s}\left(\mathbf{R}^{d}\right)$ with values in $\Gamma_{s}^{(1)}\left(\mathbf{R}^{3 d}\right)$. As a consequence, $\Psi$ satisfies

$$
\left|\partial^{\alpha} \Psi\left(x, \xi, y_{1}, y_{2}\right)\right| \lesssim h_{0}^{|\alpha|} \alpha !^{s} e^{-r_{0}\left|y_{2}\right|^{\frac{1}{s}}}
$$


for some $h_{0}, r_{0}>0$. The assertion (1) now follows from the latter estimate, Leibnitz rule and the fact that

$$
H(x, \xi, y)=v(x-y) \Psi(x, \xi, x-y)
$$

In order to prove (2) we notice that (3.9) shows that $y \mapsto H(x, \xi, x-y)$ is an element in $\mathcal{S}_{S}\left(\mathbf{R}^{d}\right)$ with values in $\Gamma_{s}^{(1)}\left(\mathbf{R}^{2 d}\right)$. By Lemma 3.6 there are $H_{2} \in C^{\infty}\left(\mathbf{R}^{3 d}\right)$ and $\phi_{0} \in \mathcal{S}_{S}\left(\mathbf{R}^{d}\right)$ such that (3.9) holds for some $h_{0}, r_{0}>0$ with $H_{2}$ in place of $H$, and

$$
H(x, \xi, x-y)=H_{2}(x, \xi, x-y) \phi_{0}(-y) .
$$

This is the same as (2), and the result follows.

Proof of Theorem 3.5 We may assume that $A=0$. Let $g=\mathrm{Op}(a) f$. By Lemma 3.7 we have

$$
\begin{aligned}
V_{\phi} g(x, \xi) & =(2 \pi)^{-\frac{d}{2}} \mathscr{F}\left(\left(f \cdot \overline{\phi_{0}(\cdot-x)}\right) \cdot H_{0}(x, \xi, \cdot)\right)(\xi) \omega(x, \xi) \\
& =(2 \pi)^{-d} \mathscr{F}\left(\left(f \cdot \overline{\phi_{0}(\cdot-x)}\right)\right) *\left(\mathscr{F}\left(H_{0}(x, \xi, \cdot)\right)\right)(\xi) \omega(x, \xi) \\
& =(2 \pi)^{-d}\left(\left(V_{\phi_{0}} f\right)(x, \cdot) *\left(\mathscr{F}\left(H_{0}(x, \xi, \cdot)\right)\right)\right)(\xi) \omega(x, \xi)
\end{aligned}
$$

Since $\omega$ and $\omega_{0}$ belongs to $\mathscr{P}_{E, s}^{0}\left(\mathbf{R}^{2 d}\right),(2)$ in Lemma 3.7 gives

$$
\left.\left|V_{\phi} g(x, \xi) \omega_{0}(x, \xi)\right| \lesssim \mid V_{\phi_{0}} f\right)(x, \cdot) \omega(x, \cdot) \omega_{0}(x, \cdot) \mid * e^{-\frac{r_{0}}{2}|\cdot| \frac{1}{s}}
$$

Here we have used the fact that

$$
\omega(x, \xi) \omega_{0}(x, \xi) \lesssim \omega(x, \xi-\eta) \omega_{0}(x, \xi-\eta) e^{\frac{r_{0}}{2}|\eta|^{\frac{1}{s}}}
$$

By applying the $\mathscr{B}$ norm we get for some $v \in \mathscr{P}_{E, s}^{0}\left(\mathbf{R}^{d}\right)$,

$$
\begin{aligned}
& \left.\|g\|_{M\left(\omega_{0}, \mathscr{B}\right)} \lesssim \| \mid V_{\phi_{0}} f\right) \cdot \omega \cdot \omega_{0} \mid *\left(e^{-r_{0}|\cdot| \frac{1}{s}} \otimes \delta_{0}\right) \|_{\mathscr{B}} \\
& \left.\quad \leq \| V_{\phi_{0}} f\right) \cdot \omega \cdot \omega_{0}\|\mathscr{B}\| e^{-r_{0}|\cdot| \frac{1}{s}} v\left\|_{L^{1}} \asymp\right\| f \|_{M\left(\omega \cdot \omega_{0}, \mathscr{B}\right)} .
\end{aligned}
$$

This gives the result.

By similar arguments as in the proof of Theorem 3.5 and Lemma 3.7 we get the following. The details are left for the reader.

Theorem 3.8 Let $A \in \mathbf{M}(d, \mathbf{R}), s \geq 1, \omega, \omega_{0} \in \mathscr{P}_{E, s}\left(\mathbf{R}^{2 d}\right), a \in \Gamma_{0, s}^{\left(\omega_{0}\right)}\left(\mathbf{R}^{2 d}\right)$, and let $\mathscr{B}$ be an invariant $B F$-space on $\mathbf{R}^{2 d}$. Then $\mathrm{Op}_{A}(a)$ is continuous from $M\left(\omega_{0} \omega, \mathscr{B}\right)$ to $M(\omega, \mathscr{B})$. 
Lemma 3.9 Let $s \geq 1, \omega \in \mathscr{P}_{E, s}\left(\mathbf{R}^{2 d}\right), \vartheta \in \mathscr{P}_{E, s}\left(\mathbf{R}^{d}\right)$ and $v \in \mathscr{P}_{E, s}\left(\mathbf{R}^{d}\right)$ be such that $v$ is submultiplicative, $\omega \in \Gamma_{0, s}^{(\omega)}\left(\mathbf{R}^{2 d}\right)$ is $v \otimes v$-moderate, $\vartheta=v^{-\frac{1}{2}}$ and $\vartheta \in \Gamma_{0, s}^{(\vartheta)}\left(\mathbf{R}^{d}\right)$. Also let $a \in \Gamma_{0, s}^{(\omega)}\left(\mathbf{R}^{2 d}\right), f, \phi \in \Sigma_{s}\left(\mathbf{R}^{d}\right), \phi_{2}=\phi v$, and let $\Phi$ and $H$ be as in Lemma 3.7. Then (3.8) and the following hold true:

(1) $H \in C^{\infty}\left(\mathbf{R}^{3 d}\right)$ and satisfies (3.9) for every $h_{0}, r_{0}>0$;

(2) there are functions $H_{0} \in C^{\infty}\left(\mathbf{R}^{3 d}\right)$ and $\phi_{0} \in \Sigma_{S}\left(\mathbf{R}^{d}\right)$ such that (3.10) holds, and such that (3.9) holds for every $h_{0}, r_{0}>0$, with $H_{0}$ in place of $H$.

We finish the section by discussing continuity for pseudo-differential operators with symbols in $\Gamma_{s}^{\left(\omega_{0}\right)}$ or in $\Gamma_{0, s}^{\left(\omega_{0}\right)}$ when acting on quasi-Banach modulation spaces. More precisely, by straight-forward computations it follows that if $\omega, \omega_{0} \in \mathscr{P}_{E, s}\left(\mathbf{R}^{2 d}\right)$ $\left(\omega, \omega_{0} \in \mathscr{P}_{E, s}^{0}\left(\mathbf{R}^{2 d}\right)\right)$, then

$$
\frac{\omega(x, \xi)}{\omega(y, \eta) \omega_{0}(y, \eta)} \lesssim \frac{\left.e^{r\left(|\xi-\eta|^{\frac{1}{s}}+|y-x|^{\frac{1}{s}}\right.}\right)}{\omega_{0}(x, \eta)}
$$

holds for some $r>0$ (for every $r>0$ ). Hence the following result is a straight-forward consequence of Propositions 2.10 and 3.3, and Lemma 3.4. (Cf. Definition 2.9 for the definition of phase split basis.)

Theorem 3.10 Let $A \in \mathbf{M}(d, \mathbf{R}), s \geq 1, \omega, \omega_{0} \in \mathscr{P}_{E, s}^{0}\left(\mathbf{R}^{2 d}\right), \boldsymbol{p} \in(0, \infty]^{2 d}, E$ be a phase split basis of $\mathbf{R}^{2 d}$, and let $a \in \Gamma_{s}^{\left(\omega_{0}\right)}\left(\mathbf{R}^{2 d}\right)$. Then $\mathrm{Op}_{A}($ a) is continuous from $M\left(L_{E}^{p}\left(\mathbf{R}^{2 d}\right), \omega_{0} \omega\right)$ to $M\left(L_{E}^{p}\left(\mathbf{R}^{2 d}\right), \omega\right)$.

The same holds true with $\mathscr{P}_{E, s}$ and $\Gamma_{0, s}^{\left(\omega_{0}\right)}$, or with $\mathscr{P}$ and $S^{\left(\omega_{0}\right)}$ in place of $\mathscr{P}_{E, s}^{0}$ and $\Gamma_{s}^{\left(\omega_{0}\right)}$, respectively, at each occurence.

Corollary 3.11 Let $A \in \mathbf{M}(d, \mathbf{R}), s \geq 1$ and $\omega, \omega_{0} \in \mathscr{P}_{E, s}^{0}\left(\mathbf{R}^{2 d}\right), p, q \in(0, \infty]$, and let $a \in \Gamma_{S}^{\left(\omega_{0}\right)}\left(\mathbf{R}^{2 d}\right)$. Then $\mathrm{Op}_{A}(a)$ is continuous from $M_{\left(\omega_{0} \omega\right)}^{p, q}\left(\mathbf{R}^{d}\right)$ to $M_{(\omega)}^{p, q}\left(\mathbf{R}^{d}\right)$.

The same holds true with $\mathscr{P}_{E, s}$ and $\Gamma_{0, s}^{\left(\omega_{0}\right)}$, or with $\mathscr{P}$ and $S^{\left(\omega_{0}\right)}$ in place of $\mathscr{P}_{E, s}^{0}$ and $\Gamma_{s}^{\left(\omega_{0}\right)}$, respectively, at each occurence.

\section{Examples}

In this section we list some examples and show how the continuity results of the pseudo-differential operators in the previous section leads to continuity on certain Sobolev spaces, weighted Lebesgue spaces and on $\Gamma_{0, s}^{(\omega)}$ spaces.

In the examples here we consider pseudo-differential operators with symbols in $\Gamma_{0, s}^{(\omega)}$ spaces. By some modifications, we may also deduce similar continuity results for operators with symbols in $\Gamma_{s}^{(\omega)}$ spaces. 
Example 4.1 Let $s \geq 1, r, r_{0} \in \mathbf{R}, A \in \mathbf{M}(d, \mathbf{R})$, and let $H_{r}^{2}\left(\mathbf{R}^{d}\right)$ be the Sobolev space of all $f \in \Sigma_{1}^{\prime}\left(\mathbf{R}^{d}\right)$ such that $\widehat{f} \in L_{l o c}^{2}\left(\mathbf{R}^{d}\right)$ and

$$
\|f\|_{H_{r}^{2}} \equiv\left(\int_{\mathbf{R}^{d}}\left|\widehat{f}(\xi) e^{r|\xi|^{\frac{1}{s}}}\right|^{2} d \xi\right)^{\frac{1}{2}}
$$

is finite. If $a \in C^{\infty}\left(\mathbf{R}^{2 d}\right)$ satisfies

$$
\left|\partial^{\alpha} a(x, \xi)\right| \lesssim h^{|\alpha|} \alpha !^{s} e^{r_{0}|\xi|^{\frac{1}{s}}}
$$

for every $h>0$, then $\mathrm{Op}_{A}(a)$ is continuous from $H_{r}^{2}\left(\mathbf{R}^{d}\right)$ to $H_{r-r_{0}}^{2}\left(\mathbf{R}^{d}\right)$.

In fact, if $\omega_{r}(x, \xi)=e^{r|\xi|^{\frac{1}{s}}} \in \mathscr{P}_{E, s}\left(\mathbf{R}^{2 d}\right)$, then it follows that the condition (4.1) holds true for every $h>0$ is the same as $a \in \Gamma_{0, s}^{\left(\omega_{r_{0}}\right)}\left(\mathbf{R}^{2 d}\right)$. By a straight-forward applications of Fourier's inversion formula we also have $H_{r}^{2}\left(\mathbf{R}^{d}\right)=M_{\left(\omega_{r}\right)}^{2,2}\left(\mathbf{R}^{d}\right)$ (cf. the proof of [17, Proposition 11.3.1]). The assertion now follows from these observations and letting $\mathscr{B}=L^{2}\left(\mathbf{R}^{2 d}\right)$ in Theorem 3.8.

Example 4.2 Let $s \geq 1, r, r_{0} \in \mathbf{R}, A \in \mathbf{M}(d, \mathbf{R})$, and let $L_{r}^{2}\left(\mathbf{R}^{d}\right)$ be the set $L_{r}^{2}\left(\mathbf{R}^{d}\right)$ which consists of all $f \in L_{l o c}^{2}\left(\mathbf{R}^{d}\right)$ such that

$$
\|f\|_{L_{r}^{2}} \equiv\left(\int_{\mathbf{R}^{d}}\left|f(x) e^{r|x|^{\frac{1}{s}}}\right|^{2} d x\right)^{\frac{1}{2}}
$$

is finite. If $a \in C^{\infty}\left(\mathbf{R}^{2 d}\right)$ satisfies

$$
\left|\partial^{\alpha} a(x, \xi)\right| \lesssim h^{|\alpha|} \alpha !^{s} e^{r_{0}|x|^{\frac{1}{s}}}
$$

for every $h>0$, then $\mathrm{Op}_{A}(a)$ is continuous from $L_{r}^{2}\left(\mathbf{R}^{d}\right)$ to $L_{r-r_{0}}^{2}\left(\mathbf{R}^{d}\right)$.

In fact, if $\omega_{r}(x, \xi)=e^{r|x|^{\frac{1}{s}}} \in \mathscr{P}_{E, s}\left(\mathbf{R}^{2 d}\right)$, then it follows that the conditions $a$ is the same as $a \in \Gamma_{0, s}^{\left(\omega_{r_{0}}\right)}\left(\mathbf{R}^{2 d}\right)$, and that $L_{r}^{2}\left(\mathbf{R}^{d}\right)=M_{\left(\omega_{r}\right)}^{2,2}\left(\mathbf{R}^{d}\right)$ (cf. the proof of [17, Proposition 11.3.1]). The assertion now follows from these observations and Theorem 3.8.

Example 4.3 Let $s \geq 1, \vartheta \in \mathscr{P}_{E, s}\left(\mathbf{R}^{2 d}\right), \vartheta_{0}=\vartheta(\cdot, 0) \in \mathscr{P}_{E, s}\left(\mathbf{R}^{d}\right), \omega_{r}$ and $\omega_{0}$ be the same as in Proposition 3.3, $A \in \mathbf{M}(d, \mathbf{R})$, and let $a \in \Gamma_{0, s}^{(\vartheta)}\left(\mathbf{R}^{2 d}\right)$. Then $\mathrm{Op}_{A}(a)$ is continuous from $\Gamma_{0, s}^{\left(\omega_{0}\right)}\left(\mathbf{R}^{d}\right)$ to $\Gamma_{0, s}^{\left(\omega_{0} \vartheta_{0}\right)}\left(\mathbf{R}^{d}\right)$.

In fact, by Theorem 3.8 it follows that

$$
\mathrm{Op}_{A}(a): M_{\left(1 / \omega_{r}\right)}^{\infty, 1}\left(\mathbf{R}^{d}\right) \rightarrow M_{\left(1 /\left(\omega_{r} \vartheta\right)\right)}^{\infty, 1}\left(\mathbf{R}^{d}\right)
$$

is continuous. Since $M_{\left(1 / \omega_{r}\right)}^{\infty, 1}\left(\mathbf{R}^{d}\right)$ is decreasing with respect to $r$ and that

$$
\omega_{r-r_{0}} \vartheta_{0} \lesssim \omega_{r} \vartheta \lesssim \omega_{r+r_{0}} \vartheta_{0}
$$


for some fixed $r_{0} \geq 0$, Proposition 3.3 shows that

$$
\bigcap_{r>0} M_{\left(1 /\left(\omega_{r}\right)\right)}^{\infty, 1}\left(\mathbf{R}^{d}\right)=\Gamma_{0, s}^{\left(\omega_{0}\right)}\left(\mathbf{R}^{d}\right) \text { and } \bigcap_{r>0} M_{\left(1 /\left(\omega_{r} \vartheta\right)\right)}^{\infty, 1}\left(\mathbf{R}^{d}\right)=\Gamma_{0, s}^{\left(\omega_{0} \vartheta_{0}\right)}\left(\mathbf{R}^{d}\right)
$$

The asserted continuity now follows from these intersections and (4.2).

Acknowledgements The author is grateful to Ahmed Abdeljawad for careful reading of the manuscript and giving valuable comments, leading to improvements of the content and the style.

Open Access This article is distributed under the terms of the Creative Commons Attribution 4.0 International License (http://creativecommons.org/licenses/by/4.0/), which permits unrestricted use, distribution, and reproduction in any medium, provided you give appropriate credit to the original author(s) and the source, provide a link to the Creative Commons license, and indicate if changes were made.

\section{References}

1. Abdeljawad, A., Cappiello, M., Toft, J.: Pseudo-differential calculus in anisotropic Gelfand-Shilov setting. Preprint. arXiv:1805.03497 (2018)

2. Abdeljawad, A., Coriasco, S., Toft, J.: Liftings for ultra-modulation spaces, and one-parameter groups of Gevrey type pseudo-differential operators. Preprint. arXiv:1712.04338 (2017)

3. Aoki, T.: Locally bounded linear topological spaces. Proc. Imp. Acad. Tokyo 18, 588-594 (1942)

4. Cappiello, M.: Pseudodifferential parametrices of infinite order for SG-hyperbolic problems. Rend. Sem. Mat. Univ. Pol. Torino 61, 411-441 (2003)

5. Cappiello, M., Toft, J.: Pseudo-differential operators in a Gelfand-Shilov setting. Math. Nachr. 290, 738-755 (2017)

6. Chung, J., Chung, S.-Y., Kim, D.: Characterizations of the Gelfand-Shilov spaces via Fourier transforms. Proc. Am. Math. Soc. 124, 2101-2108 (1996)

7. Feichtinger, H.G.: Banach spaces of distributions of Wiener's type and interpolation. In: Butzer, P., Nagy, B.S., Görlich, E. (eds.) Proceedings Conference Oberwolfach, Functional Analysis and Approximation, August 1980, Int. Ser. Num. Math., vol. 69, pp. 153-165. Birkhäuser, Basel (1981)

8. Feichtinger, H.G.: Banach convolution algebras of Wiener's type. In: Proceedings Functions, Series, Operators in Budapest, Colloquia Math. Soc. J. Bolyai, North Holland Publ. Co., Amsterdam (1980)

9. Feichtinger, H.G.: Modulation spaces on locally compact abelian groups. Technical Report, University of Vienna, Vienna, (1983); also. In: Krishna, M., Radha, R., Thangavelu, S. (eds.) Wavelets and Their Applications, pp. 99-140. Allied Publishers Private Limited, New Delhi (2003)

10. Feichtinger, H.G.: Wiener amalgams over Euclidean spaces and some of their applications. Function Spaces (Edwardsville. IL, 1990), Lecture Notes in Pure and Applied Mathematics, vol. 136, pp. 123137. Marcel Dekker, New York (1992)

11. Feichtinger, H.G.: Modulation spaces: looking back and ahead. Sample Theory Signal Image Process. 5, 109-140 (2006)

12. Feichtinger, H.G., Gröchenig, K.H.: Banach spaces related to integrable group representations and their atomic decompositions. I. J. Funct. Anal. 86, 307-340 (1989)

13. Feichtinger, H.G., Gröchenig, K.H.: Banach spaces related to integrable group representations and their atomic decompositions. II. Monatsh. Math. 108, 129-148 (1989)

14. Feichtinger, H.G., Gröchenig, K.H.: Gabor frames and time-frequency analysis of distributions. J. Funct. Anal. 146(2), 464-495 (1997)

15. Galperin, Y.V., Samarah, S.: Time-frequency analysis on modulation spaces $M_{m}^{p, q}, 0<p, q \leq \infty$. Appl. Comput. Harmon. Anal. 16, 1-18 (2004)

16. Gelfand, I.M., Shilov, G.E.: Generalized Functions, vol. I-III. Academic, New York (1968)

17. Gröchenig, K.H.: Foundations of Time-Frequency Analysis. Birkhäuser, Boston (2001)

18. Gröchenig, K.H.: Weight functions in time-frequency analysis. In: Rodino, L., Wong, M.W. (Eds.) Pseudodifferential Operators: Partial Differential Equations and Time-Frequency Analysis, Fields Institute Comm. 52, pp. 343-366 (2007) 
19. Gröchenig, K.H., Leinert, M.: Wiener's lemma for twisted convolution and Gabor frames. J. Am. Math. Soc. 17(1), 1-18 (2004)

20. Gröchenig, K.H., Zimmermann, G.: Spaces of test functions via the STFT. J. Funct. Spaces Appl. 2, 25-53 (2004)

21. Hörmander, L.: The Analysis of Linear Partial Differential Operators, vols. I-III. Springer, Berlin (1983-1985)

22. Pfeuffer, C., Toft, J.: Compactness properties for modulation spaces. Preprint. arXiv:1804.00948

23. Pilipovic, S., Prangoski, B., Vindas, J.: Translation-modulation invariant banach spaces of ultradistributions. J. Fourier Anal. Appl. (2018). https://doi.org/10.1007/s00041-018-9610-x

24. Pilipović, S., Teofanov, N.: On a symbol class of elliptic pseudodifferential operators. Bull. Acad. Serbe Sci. Arts 27, 57-68 (2002)

25. Pilipović, S., Teofanov, N.: Pseudodifferential operators on ultra-modulation spaces. J. Funct. Anal. 208, 194-228 (2004)

26. Polyanin, A.D.: Handbook of Linear Partial Differential Equations for Engineers and Scientists. Chapman \& Hall, Boca Raton (2002)

27. Rolewicz, S.: On a certain class of linear metric spaces. Bull. Acad. Polon. Sci. Sér. Sci. Math. Astrono. Phys. 5, 471-473 (1957)

28. Ruzhansky, M., Sugimoto, M., Tomita, N., Toft, J.: Changes of variables in modulation and Wiener amalgam spaces. Math. Nachr. 284, 2078-2092 (2011)

29. Tachizawa, K.: The boundedness of pseudo-differential operators on modulation spaces. Math. Nachr. 168, 263-277 (1994)

30. Teofanov, N.: Ultramodulation Spaces and Pseudodifferential Operators. Endowment Andrejević, Beograd (2003)

31. Teofanov, N.: Modulation spaces, Gelfand-Shilov spaces and pseudodifferential operators. Sample Theory Signal Image Process. 5, 225-242 (2006)

32. Toft, J.: Pseudo-differential operators with smooth symbols on modulation spaces. Cubo 11(4), 87-107 (2009)

33. Toft, J.: The Bargmann transform on modulation and Gelfand-Shilov spaces, with applications to Toeplitz and pseudo-differential operators. J. Pseudo Differ. Oper. Appl. 3(2), 145-227 (2012)

34. Toft, J.: Gabor analysis for a broad class of quasi-Banach modulation spaces. In: Pilipović, S., Toft, J. (eds.) Pseudo-Differential Operators, Generalized Functions, Operator Theory: Advances and Applications, vol. 245, pp. 249-278. Birkhäuser, Boston (2015)

35. Toft, J.: Images of function and distribution spaces under the Bargmann transform. J. Pseudo Differ. Oper. Appl. 8, 83-139 (2017)

36. Toft, J.: Continuity and compactness for pseudo-differential operators with symbols in quasi-Banach spaces or Hörmander classes. Anal. Appl. 15, 353-389 (2017)

37. Toft, J., Nabizadeh, E.: Periodic distributions and periodic elements in modulation spaces. Adv. Math. 323, 193-225 (2018)

Publisher's Note Springer Nature remains neutral with regard to jurisdictional claims in published maps and institutional affiliations. 